\title{
DAVID J.A. ROSS, ALISON STONES, MAUD PÉREZ-SIMON, Illustrated Medieval Alexander-Books in French Verse
}

\section{Graziella Pastore}

\section{(2) OpenEdition}

\section{Journals}

\section{Édition électronique}

URL : https://journals.openedition.org/studifrancesi/22182

DOI : $10.4000 /$ studifrancesi.22182

ISSN : 2427-5856

\section{Éditeur}

Rosenberg \& Sellier

\section{Édition imprimée}

Date de publication : 1 avril 2020

Pagination : 143-144

ISSN : 0039-2944

\section{Référence électronique}

Graziella Pastore, «DAVID J.A. ROSS, ALISON STONES, MAUD PÉREZ-SIMON, Illustrated Medieval Alexander-Books in French Verse », Studi Francesi [En ligne], 190 (LXIV | I) | 2020, mis en ligne le 01 avril 2020, consulté le 03 août 2021. URL : http://journals.openedition.org/studifrancesi/22182 ; DOI : https://doi.org/ 10.4000/studifrancesi.22182

Ce document a été généré automatiquement le 3 août 2021

\section{(c) (i) (9)}

Studi Francesi è distribuita con Licenza Creative Commons Attribuzione - Non commerciale - Non opere derivate 4.0 Internazionale. 


\title{
DAVID J.A. ROSS, ALISON STONES, MAUD PÉREZ-SIMON, Illustrated Medieval Alexander-Books in French Verse
}

\author{
Graziella Pastore
}

\section{RÉFÉRENCE}

DAVID J.A. ROSS, ALISON STONES, MAUD PÉREZ-SIMON, Illustrated Medieval Alexander-Books in French Verse, Turnhout, Brepols, 2019, «Manuscripta Illuminata» 4, 639 pp.

1 Cet ouvrage est consacré à l'étude du cycle iconographique du Roman d'Alexandre français en vers alexandrins, y comprises ses interpolations et continuations, et du Roman de toute chevalerie en vers anglo-normand de Thomas de Kent. Faisant suite aux Studies in Illustrated Medieval Alexander - Books in Germany and the Netherlands (Cambridge, MHRA, 1971) et aux Studies in the Alexander Romance (London, Pindar Press, 1985), le volume est le résultat de la mise au propre des derniers travaux de David J.A. Ross sur la tradition iconographique de l'Alexandre en vers français. Les AA., A. Stones et M. Pérez-Simon, tout en essayant de respecter les intentions de Ross (choix d'édition, p. 10), ont édité et amendé les textes manuscrits et tapuscrits laissés inachevés, effectué les vérifications nécessaires sur les manuscrits cités, intégré les descriptions et les attributions stylistiques, et complété l'ouvrage par des références actualisées et d'utiles tables comparatives. On ne peut que les féliciter d'avoir introduit une section importante de reproductions en couleurs, ce qui facilite la lisibilité de l'ensemble, tout en permettant l'accès aux manuscrits non disponibles en ligne.

2 Le volume s'articule en deux grandes parties: The "Alexandre de Paris" Tradition (pp. 11-222) et The Thomas de Kent Tradition (pp. 223-405); elles sont complétées par un riche apparat figuratif (pp. 459-639) contenant notamment plusieurs reproductions du manuscrit B du Roman d'Alexandre, composé dans l'Italie du Nord vers la fin du XIII ${ }^{\mathrm{e}}$ ou le début du XIV e s. (Venise, Museo Correr 1493). 
3 Après une introduction qui délimite le champ d'étude et résume efficacement les sources et l'histoire de la transmission du Roman d'Alexandre (pp.13-30), le premier chapitre de la première partie contient la description de chaque manuscrit de la tradition complétée par la description ponctuelle des illustrations et la transcription des rubriques correspondantes (The illustration of the "Roman d'Alexandre" Proper, pp.31-131); ce chapitre étudie également les relations entre les différents cycles iconographiques, efficacement explicitées par un stemma (p.122). Le deuxième chapitre porte sur l'étude des cycles iconographiques de deux des interpolations du Roman d'Alexandre: La prise de Defur et Le voyage d'Alexandre au paradis terrestre (Interpolations in the "Roman d'Alexandre", pp. 133-156). Le troisième chapitre s'intéresse aux illustrations du manuscrit Città del Vaticano, BAV, Arch. San Pietro E.36, contenant la version latine de la Fuerre de Gadres d'Eustache (MS V, The Vatican Manuscript of the Latin Prose "Fuerre de Gadres", pp.157-166). Le quatrième chapitre étudie les cycles iconographiques présents dans les manuscrits de la Venjance Alixandre de Jean de Nevelon et du Vengement Alixandre de Gui de Cambrai (The "Vengeance" Sequels, pp. 167-175). La première partie est clôturée par six appendices (pp. 167-222): 1) la description du cycle iconographique du manuscrit $\mathrm{C}^{\mathrm{m}}$ composé dans les Flandres vers 1220 (Nottingham, UL, WLC/LM 6); 2) la transcription du calendrier à l'usage de Tournai contenu dans le manuscrit $\mathrm{H}$ du Roman d'Alexandre (Paris, BnF, fr. 786, ff. 1-3v); 3) une table qui résume la description et le contenu de tous les manuscrits transmettant l'intégralité ou une partie du Roman d'Alexandre de Paris; 4) un ensemble de tables synoptiques des illustrations contenues dans les manuscrits du Roman d'Alexandre, de ses interpolations et continuations; 5) une table des interpolations organisée par manuscrit; 6) un ensemble de notes au sujet des manuscrits non illustrés et des fragments.

4 La seconde partie s'ouvre avec une introduction résumant le contenu et la tradition du Roman de toute chevalerie de Thomas de Kent ainsi que par la liste des témoins connus (pp. 225-234). Le premier chapitre est consacré à l'analyse détaillée des illustrations contenues dans chaque manuscrit, accompagnée par la transcription des rubriques correspondantes (The Picture-Cycle, pp. 235-386). Le second chapitre résume enfin les correspondances iconographiques entre les différents témoins de l'ensemble de la tradition (Iconographic correspondences between the "Roman de toute chevalerie" and Manuscripts B, C, and P, pp. 387-405).

5 Le volume est enfin complété par une brève conclusion générale (p. 407), l'index des manuscrits cités (pp. 409-413), l'index des noms propres (pp.414-428), la liste des abréviations (pp. 429-430), la bibliographie (pp. 431-448) et la liste des illustrations et des crédits photographiques (pp. 449-458). 